\title{
SOME RESULTS ON PIN AND ITO PHOTODIODES
}

\author{
M.A. GRADO CAFFARO and M. GRADO CAFFARO \\ (Received June 11, 1992; in final form August 6, 1992)
}

In this paper, various contributions concerning generalized photodiodes and ITO photodiodes are reported. In particular, calculations related to the bandwidth of the equivalent circuit of a PIN photodiode are performed and compared to experimental considerations. In addition, an ITO structure on a layer n-type GaAs is examined. An ITO-HEMT is also considered.

\section{INTRODUCTION}

For frequencies higher than a few $\mathrm{GHz}$, the best photodetector devices are PIN (or Schottky) diodes or ITO photodiodes combined with a MESFET or HEMT.

In particular, hybrid ITO-HEMT is an excellent photodetector for the millimeter wave range. In the following, we will analyze some important aspects related to generalized PIN photodiodes, ITO photodiodes and ITO-HEMTs. Certainly, ITOHEMT is a hybrid device with excellent performance in the millimeter wave range since an ITO photodiode is a good detector and HEMT is an advanced transistor which, in our context, is used as amplifier. A PIN-HEMT is also an excellent hybrid device.

\section{SOME REMARKS ON PHOTODIODE THEORY}

In the context of generation process, the number of excited electron-hole pairs is given by the formula:

$$
\delta \mathrm{n}=\mathrm{g} \tau_{\mathrm{eff}}
$$

where $\mathrm{g}$ is the generation rate and $\tau_{\text {eff }}$ the effective carrier life-time.

Assuming a generalized PIN photodiode (figure 1), it is well known that the bandwidth of a photodiode is limited by the following factors:

1) Transit time of generated electron-hole pairs in the depletion zone.

2) The active region capacitance.

3) The diffusion of slow moving carriers generated outside the active zone.

4) Stray effects originated by the connection of the device to an exterior circuit. 


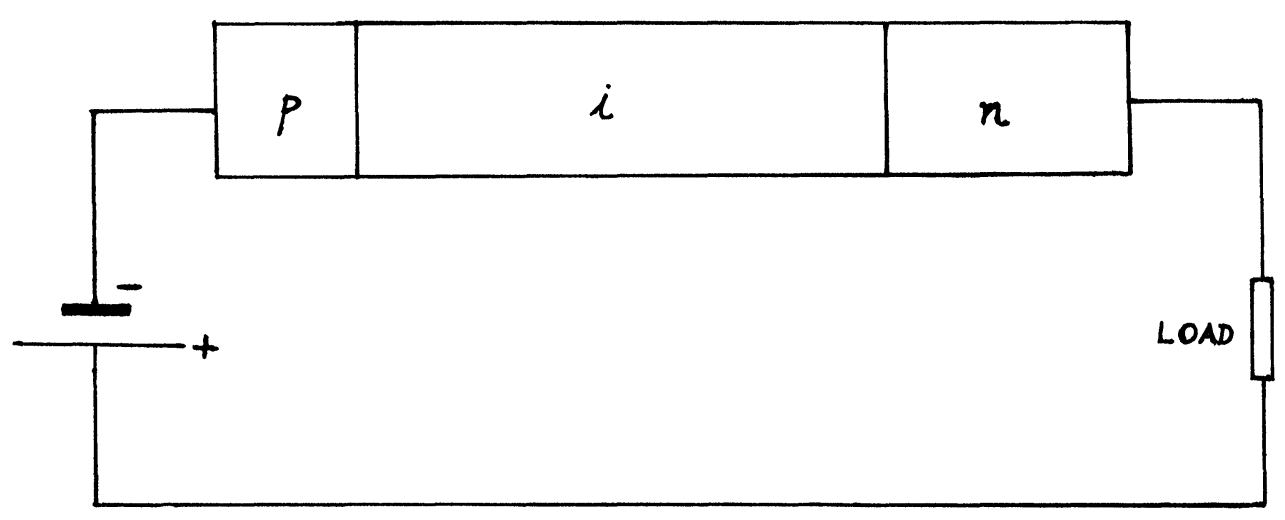

FIGURE 1 Basic PIN bias circuit.

In practice, the speed of response (or the bandwidth) of the device in question is controlled by the time constants $\tau_{\mathrm{tr}}$ and $\tau_{\mathrm{RC}}$, namely:

a) $\tau_{\mathrm{tr}}$ is a measure of the finite transit time of electron-hole pairs generated in the depletion region.

b) $\tau_{\mathrm{RC}}$ results from considering the device as a parallel plate capacitor.

Then the $-3 \mathrm{~dB}$ bandwidth corresponding to the photodiode is:

$$
\mathrm{B}=\frac{1}{\tau_{\text {eff }}}
$$

where $\tau_{\text {eff }}$ is given by the following expression: ${ }^{1}$

$$
\tau_{\mathrm{eff}} \simeq 2^{\prime} 86 \tau_{\mathrm{tr}}+2^{\prime} 26 \tau_{\mathrm{RC}}
$$

Substituting (3) into (2) we obtain:

$$
\mathrm{B}=\frac{50}{143 \tau_{\mathrm{tr}}+113 \tau_{\mathrm{RC}}}
$$

Equation (4) correlate to the research work of Wang and Bloom,${ }^{2}$ whereby a series inductance of $50 \mathrm{pH}$ was introduced (fig. 2) to peak the speed of response.

With respect to figure 2, $\mathrm{R}$ is the load resistance of the photodetector in question and $\mathrm{C}$ represents the junction capacitance of the device. Now, consider the following values:

$$
\begin{aligned}
\tau_{\mathrm{tr}} & \simeq 4 \mathrm{ps} \quad(\mathrm{a}) \\
\tau_{\mathrm{RC}} & \simeq 0.6 \mathrm{ps}(\mathrm{b}) \\
\mathrm{L} & =50 \mathrm{pH} .
\end{aligned}
$$




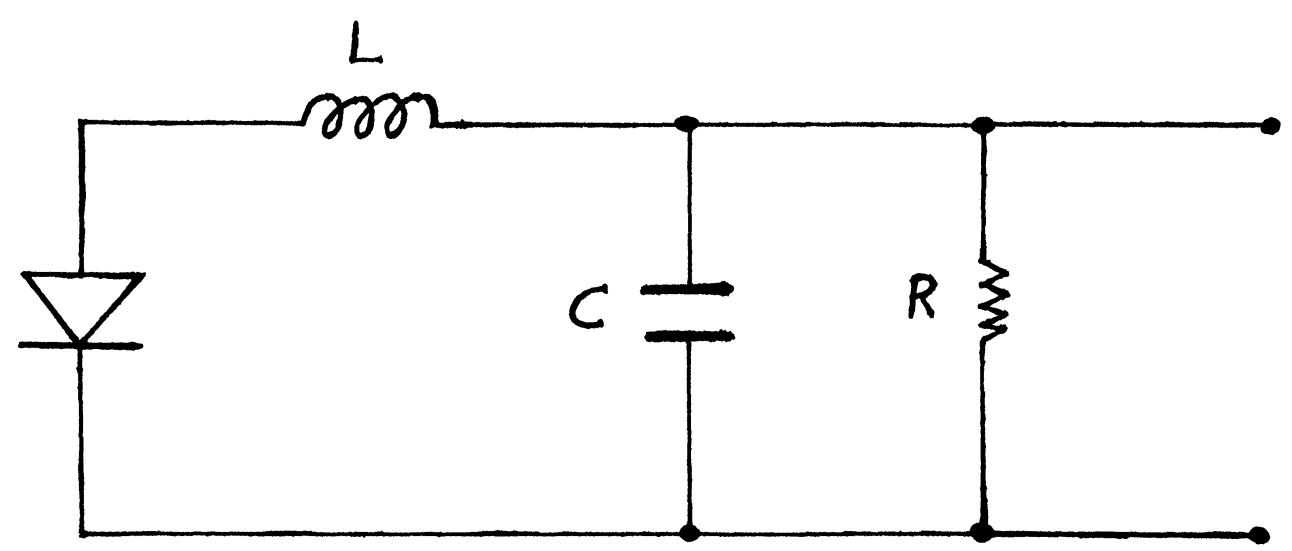

FIGURE 2 Equivalent circuit of the detector with a series coil.

By using the values (a) and (b) in equation (4), we obtain: $\mathrm{B} \simeq 78 \mathrm{GHz}$, in good agreement with Wang and Bloom. ${ }^{2}$ The above values (a) and (b) correspond to a $5 \mu \mathrm{m}$ square diode with a $0.40 \mu \mathrm{m}$ active zone.

Of interest is the calculation of the quality factor $(Q)$ from the formula:

$$
B^{\prime}=\frac{f_{0}}{Q}
$$

where $f_{0}$ is the resonance frequency, which is obtained from:

$$
\mathrm{f}_{0}=\frac{1}{2 \pi \sqrt{\mathrm{LC}}}
$$

and $\mathrm{B}^{\prime}$ is the modified bandwidth $(-3 \mathrm{~dB})$ because of the introduction of $\mathrm{L}$.

\section{ITO PHOTODIODES}

Consider a structure consisting of the compound $\operatorname{In}_{1-\mathrm{x}} \mathrm{Sn}_{\mathrm{x}} \mathrm{O}$ on a layer n-type GaAs. Moreover, consider an underlying n-type layer strongly doped in order to create a low resistance ohmic contact. In our experiment the structure in question is grown by vapor phase epitaxy. With respect to the composition of sputtered indium tin oxide, $\mathrm{x}=0.2$. A $80 \mathrm{~nm}$ layer of ITO on a $1 \mu \mathrm{m}$ thick GaAs layer is employed; this layer is doped with $\mathrm{N}_{\mathrm{D}}=10^{15} \mathrm{~cm}^{-3}$ (donor density) making the GaAs layer n-type.

$\mathrm{In}_{0.8} \mathrm{Sn}_{0.2} \mathrm{O}$ presents a wide band-gap (approximately, $3.8 \mathrm{eV}$ ) and, hence, the structure is nearly transparent at $840 \mathrm{~nm}$-wavelength. Moreover, the thickness of the ITO $(80 \mathrm{~nm})$ is adequate to function as an antireflection coating. ${ }^{3}$

On the other hand, a typical ITO presents d.c. characteristics such that a leakage current of approximately $1 \mathrm{nA}$ corresponds to a voltage of $-5.5 \mathrm{v},{ }^{1}$ by assuming a device with $28 \mathrm{v}$ of reverse breakdown voltage. 
In our research work, we have obtained the following conclusion: the strongly doped insulator involved in the device in question behaves as a degenerate material resulting in the formation of a diode. Several calculations performed by us confirm this claim. Moreover, it is feasible to obtain the previous conclusion experimentally.

With respect to the ITO/HEMT hybrid device, we have obtained a leakage current of about $1.6 \mathrm{nA}$ at $-5.2 \mathrm{v}$, using a $0.5 \mu \mathrm{m}$ gate-length HEMT with an operating point such that $\mathrm{V}_{\mathrm{DS}}=2.5 \mathrm{v}$; the frequency of operation was $8 \mathrm{GHz}$. Moreover, the $\mathrm{I}_{\mathrm{DSs}}$ was equal to $50 \mathrm{~mA}$.

\section{DISCUSSION}

We have performed a brief study on ITO photodiodes in order to establish various conclusions related to their physical structure and in combination with HEMT. In addition, considerations on bandwidth of the equivalent circuit of a generalized PIN photodiode have been exposed. Basically, these considerations are applicable to ITO devices. Also, it is easy to obtain important conclusions from the small signal equivalent circuit for an ITO/HEMT in order to estimate bandwidth, quality factor, etc., namely, the approximated equation for the effective carrier life-time is relevant in our context since this quantity is strongly related to bandwidth.

\section{REFERENCES}

1. D.G. Parker, "The fabrication, theory and assessment of Photodiodes with picosecond times". GEC J. Res. 4, 2, pp. 177-181 (1987).

2. S.Y. Wang, and D.M. Bloom, "100 GHz bandwidth planar GaAs Schottky photodiode". Elect. Lett. 19, pp. 554-555, (1983).

3. D.G. Parker, "Use of transparent Indium Tin Oxide to form a highly efficient $20 \mathrm{GHz}$ Schottky barrier photodiode". Elect. Lett., 21, pp. 778-779, (1985). 

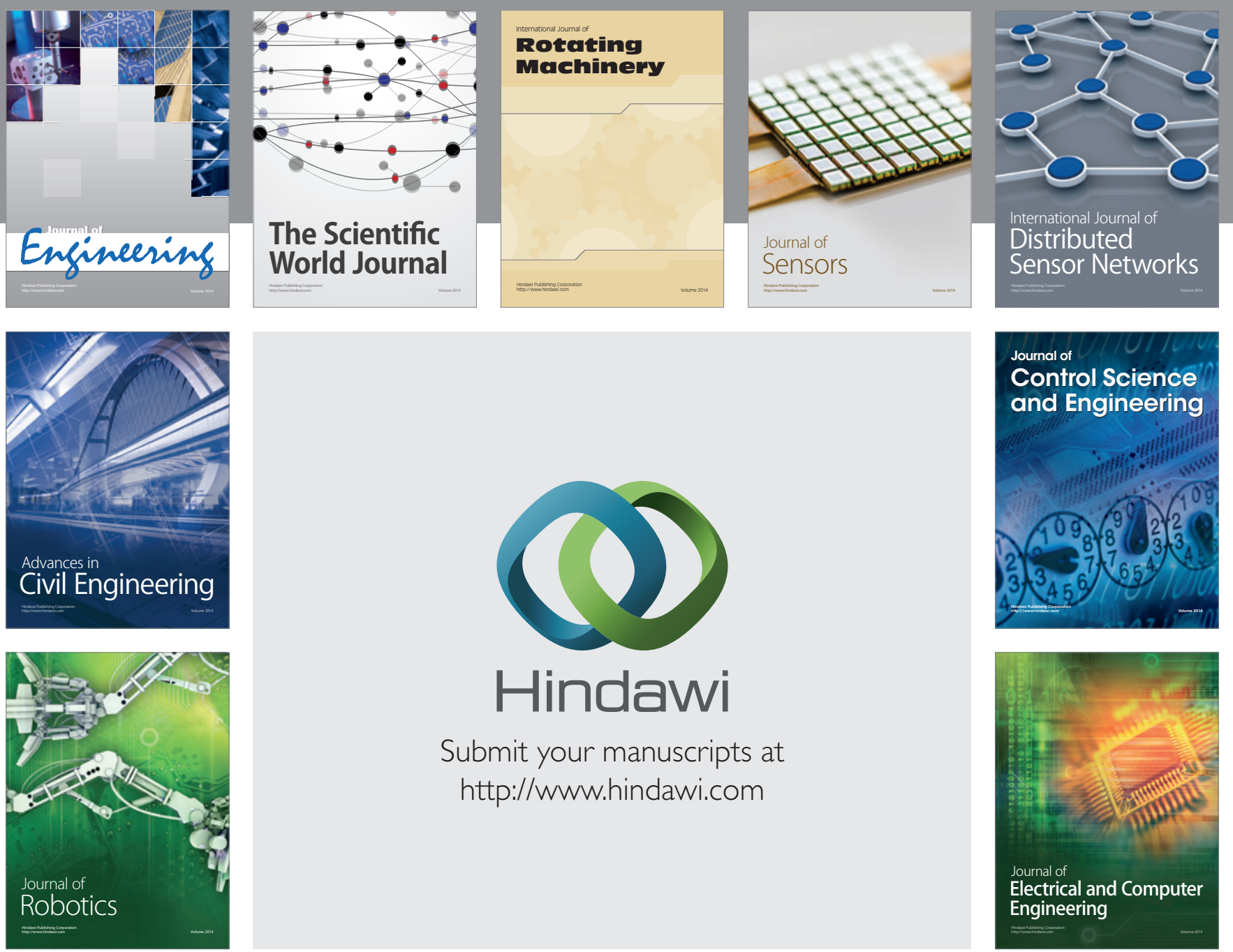

Submit your manuscripts at

http://www.hindawi.com
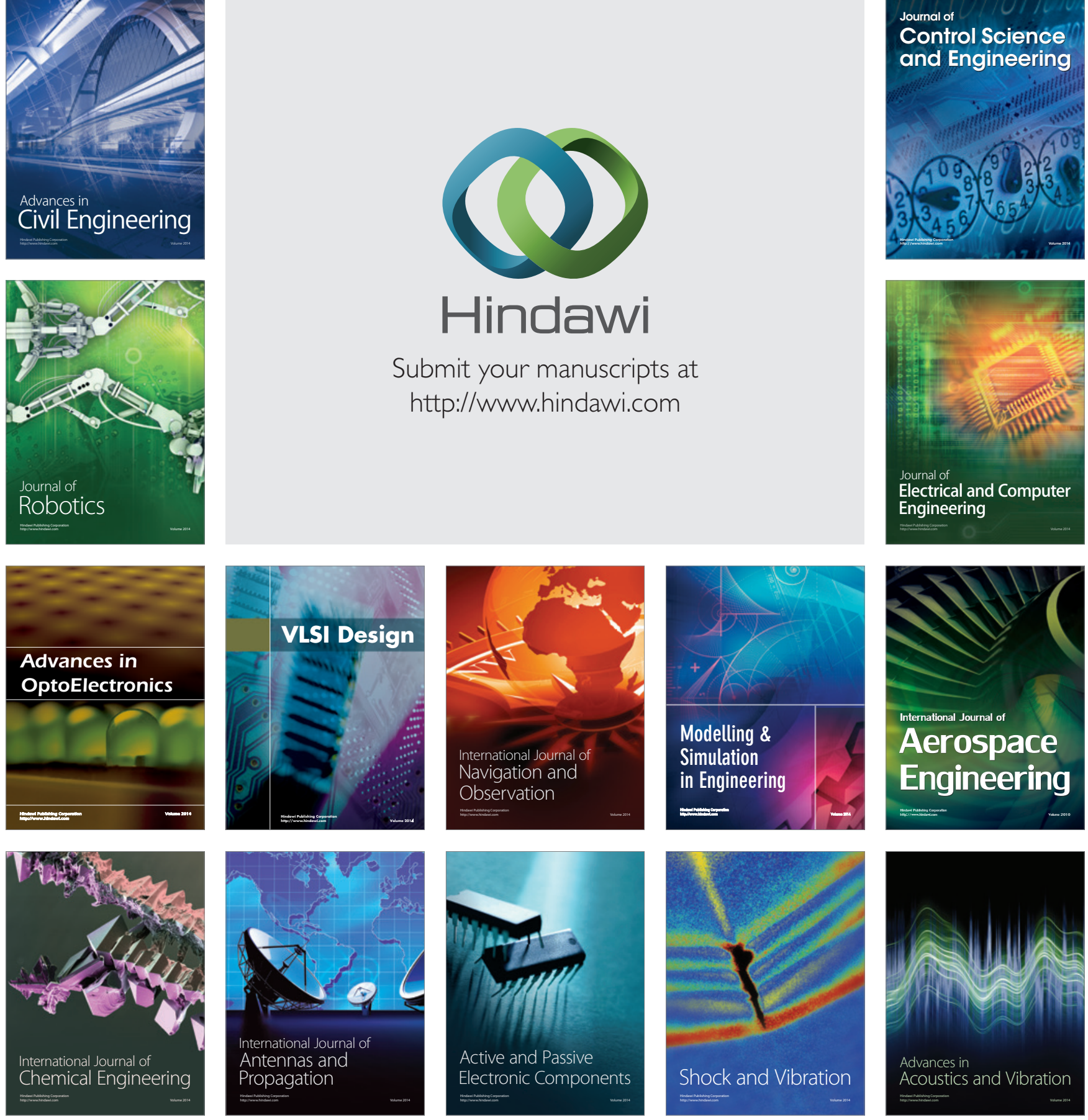